\title{
Radiation-Induced Sarcoma: A 15-Year Experience in a Single Large Tertiary Referral Center
}

\section{Kyung Su Kim, MD' \\ Ji Hyun Chang, MD² \\ Noorie Choi, MD ${ }^{1}$ \\ Han-Soo Kim, MD, PhD ${ }^{3}$ \\ Ilkyu Han, MD, PhD ${ }^{3}$ \\ Kyung Chul Moon, MD, PhD \\ II Han Kim, MD, PhD' \\ Hak Jae Kim, MD, $P h D^{1}$}

${ }^{1}$ Department of Radiation Oncology, Seoul National University College of Medicine, Seoul, ${ }^{2}$ Department of Radiation Oncology, Seoul St. Mary's Hospital,

College of Medicine,

The Catholic University of Korea, Seoul, Departments of ${ }^{3}$ Orthopedic Surgery and ${ }^{4}$ Pathology, Seoul National University

College of Medicine, Seoul, Korea

\author{
Correspondence: Hak Jae Kim, MD, PhD \\ Department of Radiation Oncology, \\ Seoul National University College of Medicine, \\ 103 Daehak-ro, Jongno-gu, Seoul 03080, Korea \\ Tel: 82-2-2072-2520 \\ Fax: 82-2-765-3317 \\ E-mail: khjae@snu.ac.kr \\ Received May 19, 2015 \\ Accepted August 17, 2015 \\ Published Online September 9, 2015 \\ ${ }^{*}$ Kyung Su Kim and Ji Hyun Chang contributed \\ equally to this work.
}

\section{Purpose}

The purpose of this study is to report on the incidence and the experience in management of radiation-induced sarcoma (RIS) at a large single center in Korea for 15 years.

\section{Materials and Methods}

We retrospectively reviewed the sarcoma registry of a large institution from January 2000 to April 2014.

\section{Results}

Out of the 3,674 patients listed in the registry, 33 patients $(0.9 \%)$ diagnosed with RIS were identified. The median latency of RIS was 12.1 years. The number of cases of RIS increased from four cases in the years 2000-2003 to 14 cases in the years 2012-2014. The most common histology was osteosarcoma (36.4\%). The median follow-up period was 23.1 months, the median overall survival (OS) of all patients was 2.9 years, and their 5-year survival rate was $44.7 \%$. Univariate and multivariate analyses showed association of the age at diagnosis $(p=0.01)$ and the treatment aim $(p=0.001)$ with the OS. The median OS and the 5 -year survival rate of patients treated with curative surgery $(n=19)$ were 9.6 years and $65 \%$, respectively, and of the conservatively treated patients, 0.7 years and $0 \%(n=14)$. Re-irradiation was delivered to nine patients, and radiation toxicity was observed in five patients.

\section{Conclusion}

In this study, RIS accounted for $0.9 \%$ of the cases of sarcoma, with increasing incidence. Despite the association of curative resection with increased survival, it could be applied to only $58 \%$ of the patients. Considering the limited treatment options for RIS, conduct of a genetic study to identify the underlying mechanism of RIS is needed.

Key words

Radiation-induced neoplasms, Sarcoma, Survival

\section{Introduction}

Radiation-induced sarcoma (RIS) is a rare late event after radiation therapy (RT), with a reported incidence of $0.03 \%$ $0.2 \%$ in 5 years and overall up to $0.8 \%[1,2]$. It is known to have a worse prognosis than sporadic soft tissue sarcoma
(STS), with a 5-year disease-specific survival (DSS) rate of $32 \%-58 \%$ in recent reports [3-5]. Gladdy et al. [3] reported that RIS had a worse DSS than sporadic STS with a hazard ratio of 1.7. Dineen et al. [6] also recently reported a lower DSS of RIS than of sporadic STS.

Because RIS arises in the previously treated area, there are many limitations in its management. The major limitation is 
that RIS has lower resectability than sporadic sarcomas. Thijssens et al. [7] emphasized the low possibility of curative resection with data showing $62 \% \mathrm{R} 0$ resection. In the study by Neuhaus et al. [8], R0-1 resection was achieved in approximately $37 \%$ of the patients and was the only factor showing significant correlation with the DSS. In addition, adjuvant RT for RIS should be administered with caution because of the higher risk of RT-related toxicities.

RT plays a critical role in the treatment of most cancers. Therefore, concerns about RIS are emerging among cancer survivors because the survival rate and the survival time after cancer treatment are increasing as a result of advanced treatments including the use of targeted agents.

Most studies of RIS in Asia are case reports, and significantly under-reported compared to western countries. RIS patients tend to be referred to a large tertiary center because of the surgical complexity, therefore we report herein the incidence and experience in management of RIS at a single large tertiary referral center for 15 years.

\section{Materials and Methods}

After receiving institutional review board approval, we retrospectively reviewed our institutional sarcoma registry from January 2000 to April 2014. Out of the 3,674 patients in the registry, we identified 33 patients who met the following criteria for RIS, which were modifications of Cahan's criteria: (1) pathologic confirmation of sarcoma that was histologically unique from the primary cancer, (2) occurrence of the sarcoma in the field of radiation, and (3) radiation exposure at least 6 months before development of the sarcoma [9].

Medical records were reviewed for the patients' age, sex, primary cancer treatment, RIS characteristics, RIS treatment, and follow-up data. The radiographic image of RIS and the histopathology report were assessed for identification of the tumor location, size, and histology. All available medical records from the follow-up were reviewed for evaluation of the disease status and toxicity. The toxicities from re-irradiation were evaluated using the Common Terminology Criteria for Adverse Events (CTCAE) ver. 4.0.

\section{Statistical analysis}

The overall survival rate was calculated from the time of the RIS diagnosis until death. Actuarial survival curves were plotted from the RIS diagnosis using the Kaplan-Meier method. A p-value less than 0.05 was considered to indicate a statistically significant difference. Analyses were performed using PASW ver. 18.0 (SPSS Inc., Chicago, IL).

\section{Results}

\section{Patients and tumor characteristics}

A total of 33 patients diagnosed with RIS were identified. The number of cases increased from four cases in the years 2000-2003 to 14 cases in the years 2012-2014 with corresponding proportion of RIS among sarcoma patients of $0.5 \%$ to 1.6\% (Fig. 1).

A list of the characteristics of the 33 patients is shown in Table 1. Females accounted for $75.8 \%$ of the patients. The median age at the primary radiotherapy was 44 years (range, 0 to 71 years). Three patients were treated before age 20 (for bilateral retinoblastoma, Wilms' tumor, and nasopharyngeal cancer), and 20 patients were treated in their 40s and 50s. The median age at the RIS diagnosis was 55 years (range, 22 to 85 years), and the median latency was 12.1 years (range, 1.9 to 28.8 years).

The most common type of primary cancer was breast cancer $(n=9)$, followed by uterine cervical cancer $(n=8)$, and head and neck cancer $(\mathrm{n}=7)$. The median prescribed dose for primary RT was 50.4 Gy. All patients received a dose greater than $45 \mathrm{~Gy}$, with the exception of three patients with Wilms' tumor, retinoblastoma, and lymphoma treated with $10.8 \mathrm{~Gy}$, $36 \mathrm{~Gy}$, and $30 \mathrm{~Gy}$, respectively. Four patients underwent intracavitary brachytherapy in addition to external-beam RT for treatment of cervical cancer; $58 \%$ of patients underwent chemotherapy as their primary cancer treatment.

RIS was located in the trunk in $75.8 \%$ of the cases, and in the head and neck in $24.2 \%$. The most common histology was osteosarcoma (36.4\%), followed by malignant fibrous histio-

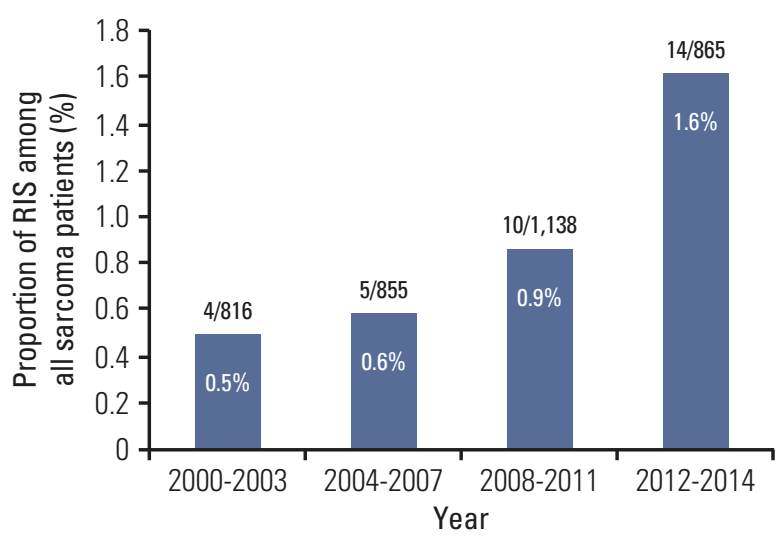

Fig. 1. Incidence of radiation induced sarcoma in a tertiary referral center in Korea. Numbers in parentheses denote the radiation-induced sarcoma (RIS) cases per all sarcoma patients in given periods. 
cytoma (33.3\%) and angiosarcoma (6.1\%). The median tumor size was $4.8 \mathrm{~cm}$ (range, 1.5 to $12.5 \mathrm{~cm}$ ), and tumors were located deep in the muscle fascia in 27 cases $(81.8 \%)$.

\section{Treatment of RIS and outcome}

The median follow-up period was 23.1 months (range, 2.2 to 174.8 months). Nineteen patients underwent surgery with curative intent, while 14 patients were treated conservatively. A list of the details of the treatment of 19 patients with curative surgery is shown in Table 2. R0 resection was achieved, except in one patient. Four patients underwent neo-adjuvant chemotherapy, and six patients, adjuvant chemotherapy. One patient diagnosed with malignant histiocytoma in the mandible underwent adjuvant RT. The median overall survival period of all patients was 2.9 years, and their 5-year survival rate was $44.7 \%$. In univariate and multivariate analyses, the age at diagnosis and the treatment aim showed association with the overall survival (Table 3). The median duration of the overall survival period and the 5-year survival rate of patients treated with curative surgery were 9.6 years and $65 \%$, respectively, and that of the conservatively treated patients, 0.7 years and $0 \%(\mathrm{p}=0.001)$ (Fig. 2).

The treatment outcomes are summarized in Fig. 3. Out of the 19 patients treated with curative resection, local recurrence occurred in nine patients $(47 \%)$, and distant metastasis, in seven patients $(37 \%)$.

\section{Re-irradiation and toxicity}

Nine patients underwent re-irradiation for various intents. Four patients were misdiagnosed with bone metastasis from their primary tumor and underwent palliative RT before biopsy. Three patients underwent palliative radiotherapy, one patient was treated with gamma knife surgery of the recurred mass, and one patient underwent adjuvant $\mathrm{RT}$ after curative surgery. Five patients, including the familial retinoblastoma patient who developed grade 4 skin necrosis after re-irradiation, showed radiation toxicity after re-irradiation (Table 4).

\section{Discussion}

To the best of our knowledge, this is the first study reporting on the incidence and survival of RIS in Asia in a significant number of patients. Our data showed that, although rare, the number of patients diagnosed with RIS in our institution is consistently increasing with time. This may be due to the significant increase of RT usage in cancer therapy in
Table 1. Characteristics of patients and radiation-induced sarcoma (RIS)

\begin{tabular}{|c|c|}
\hline Characteristic & No. $(\%)$ \\
\hline \multicolumn{2}{|l|}{ Sex } \\
\hline Male & $8(24.2)$ \\
\hline Female & $25(75.8)$ \\
\hline Age at primary radiotherapy, median (range, yr) & $44(0-71)$ \\
\hline $0-19$ & $3(9.1)$ \\
\hline $20-39$ & $6(18.2)$ \\
\hline $40-59$ & $20(60.6)$ \\
\hline$\geq 60$ & $4(12.1)$ \\
\hline Age at diagnosis of RIS, median (range, yr) & $55(22-85)$ \\
\hline Latency, median (range, yr) & $12.1(1.9-28.8)$ \\
\hline \multicolumn{2}{|l|}{ Primary tumor } \\
\hline Breast ca. & $9(27.3)$ \\
\hline Cervical ca. & $8(24.2)$ \\
\hline Rectal ca. & $5(15.2)$ \\
\hline Head and neck ca. & $7(21.2)$ \\
\hline Retinoblastoma & $1(3.0)$ \\
\hline Craniopharyngioma & $1(3.0)$ \\
\hline Lymphoma (DLBC, spine) & $1(3.0)$ \\
\hline Wilms' tumor & $1(3.0)$ \\
\hline \multicolumn{2}{|l|}{ Radiation dose (Gy) } \\
\hline$<20$ & $1(3.0)$ \\
\hline $20-40$ & $2(6.0)$ \\
\hline $40-60$ & $17(51.5)$ \\
\hline$\geq 60^{\mathrm{a})}$ & $13(39.4)$ \\
\hline \multicolumn{2}{|l|}{ Chemotherapy for primary cancer } \\
\hline Yes & $21(63.3)$ \\
\hline No & $12(36.7)$ \\
\hline \multicolumn{2}{|l|}{ RIS location } \\
\hline Trunk & $25(75.8)$ \\
\hline Head and neck & $8(24.2)$ \\
\hline \multicolumn{2}{|l|}{ RIS histology } \\
\hline Osteosarcoma & $12(36.4)$ \\
\hline MFH & $11(33.3)$ \\
\hline Angiosarcoma & $2(6.1)$ \\
\hline Chondrosarcoma & $1(3.0)$ \\
\hline Endometrial stromal sarcoma & $1(3.0)$ \\
\hline Low-grade fibromyxoid sarcoma & $1(3.0)$ \\
\hline Myxofibrosarcoma & $1(3.0)$ \\
\hline Myxoid liposarcoma & $1(3.0)$ \\
\hline Undifferentiated sarcoma & $1(3.0)$ \\
\hline Sarcoma unspecified & $1(3.0)$ \\
\hline Tumor size, median (range, cm) & $4.8(1.5-12.5)$ \\
\hline \multicolumn{2}{|l|}{ Tumor depth } \\
\hline Deep & $27(81.8)$ \\
\hline Superficial & $6(18.2)$ \\
\hline
\end{tabular}

RIS, radiation induced sarcoma; DLBC, diffuse large B cell; $\mathrm{MFH}$, malignant histiocytoma. ${ }^{\mathrm{a}}$ Four patients received intracavitary brachytherapy in addition to external-beam radiation therapy for treatment of cervical cancer. 


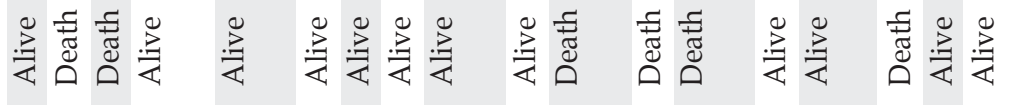

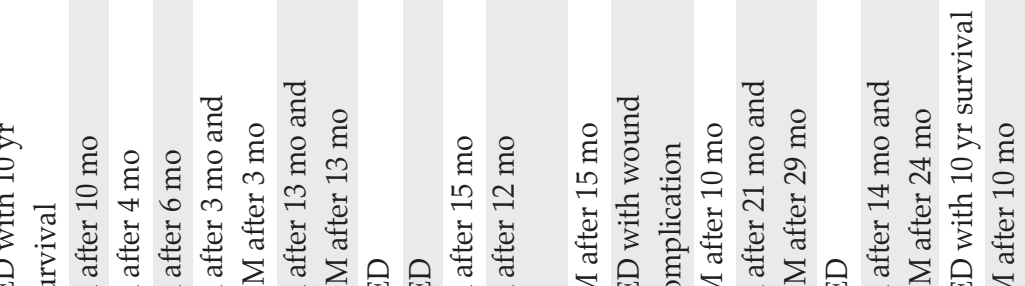

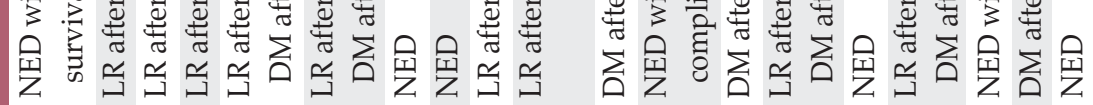

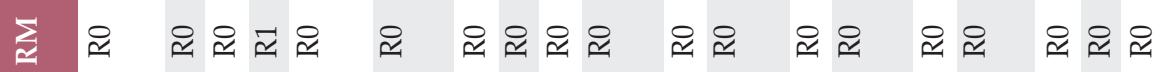

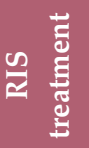

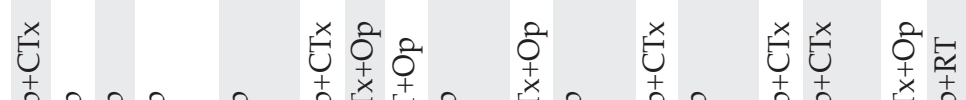

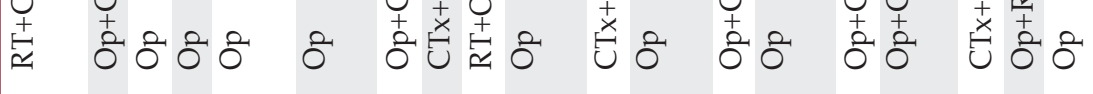

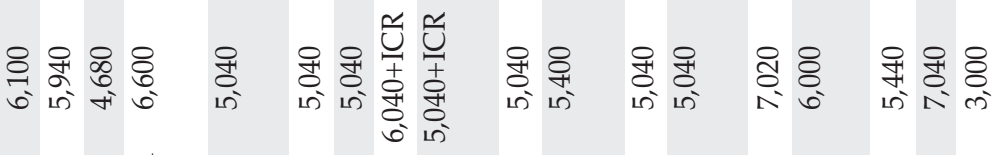
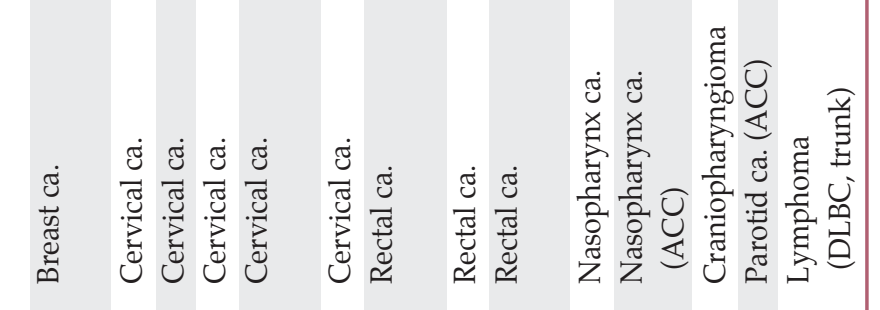

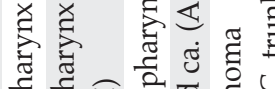

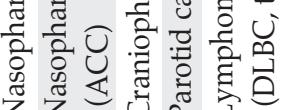


Table 3. Univariate and multivariate analyses for overall survival

\begin{tabular}{|c|c|c|c|c|}
\hline Characteristic & No. & $\begin{array}{c}\text { Median } \\
\text { survival (mo) }\end{array}$ & $\begin{array}{c}\text { p-value }{ }^{a)} \\
\text { (univariate) }\end{array}$ & $\begin{array}{c}\mathrm{p} \text {-value } \mathrm{e}^{\mathrm{b})} \\
\text { (multivariate) }\end{array}$ \\
\hline \multicolumn{5}{|l|}{ Sex } \\
\hline Male & 8 & 28.4 & 0.601 & 0.204 \\
\hline Female & 25 & 23.1 & & \\
\hline \multicolumn{5}{|c|}{ Age at primary radiotherapy, median (yr) } \\
\hline$\leq 44$ & 17 & 34.9 & 0.886 & - \\
\hline$>44$ & 16 & 23.1 & & \\
\hline \multicolumn{5}{|c|}{ Age at diagnosis of RIS, median (yr) } \\
\hline$\leq 55$ & 18 & 115.2 & 0.218 & $0.010^{c)}$ \\
\hline$>55$ & 15 & 23.1 & & \\
\hline \multicolumn{5}{|l|}{ Latency, median } \\
\hline$\leq 12$ & 16 & 115.2 & 0.233 & 0.176 \\
\hline$>12$ & 17 & 23.3 & & \\
\hline \multicolumn{5}{|c|}{ Chemotherapy for primary cancer } \\
\hline Yes & 21 & 34.9 & 0.704 & - \\
\hline No & 12 & 28.4 & & \\
\hline \multicolumn{5}{|l|}{ RIS location } \\
\hline Trunk & 25 & 34.9 & 0.613 & 0.865 \\
\hline Head and neck & 8 & 14.8 & & \\
\hline \multicolumn{5}{|l|}{ RIS histology } \\
\hline MFH & 11 & 124.0 & 0.121 & 0.376 \\
\hline Osteosarcoma & 12 & 23.3 & & \\
\hline Others & 10 & 14.8 & & \\
\hline \multicolumn{5}{|l|}{ Tumor size $(\mathrm{cm})$} \\
\hline$\leq 5$ & 19 & 34.9 & 0.858 & 0.284 \\
\hline$>5$ & 14 & 23.3 & & \\
\hline \multicolumn{5}{|l|}{ Tumor depth } \\
\hline Deep & 27 & 22.2 & 0.980 & 0.320 \\
\hline Superficial & 6 & 34.9 & & \\
\hline \multicolumn{5}{|l|}{ Treatment } \\
\hline Curative & 19 & 115.2 & 0.001 & $0.001^{\mathrm{c})}$ \\
\hline Palliation & 14 & 8.0 & & \\
\hline
\end{tabular}

RIS, radiation induced sarcoma; MFH, malignant histiocytoma. ${ }^{a}$ Log-rank test, ${ }^{b}$ Cox regression analysis, ${ }^{\mathrm{c}}$ Statistically significant.

South Korea since the first Cobalt-60 teletherapy installation in 1963. Patients undergoing RT increased from 22,000 in 1999 to 52,000 in 2011 [10]. The increased survival time of cancer patients could also be attributed to the increasing incidence of RIS.

RIS is known to have a worse prognosis than sporadic STS. Bjerkehagen et al. [5] investigated the question of whether or not a previous RT history is a worse prognosticator. In their analysis, a previous RT history was not a prognostic factor, but unlike sporadic STS, RIS tended to occur in a central location and to be associated with incomplete surgery, which are known poor prognosticators in sarcoma. As Cha et al. [11] stated, previous RT might obscure the anatomic and tumor planes, which might make radical resection challeng- ing. Consistently in our data, only $57.6 \%$ of the patients could be treated with curative intent, and the survival outcome differed significantly according to the treatment aim.

In addition, the adjuvant RT for RIS is limited by the concern for possible complications regarding re-irradiation, which can hinder optimal RIS management. An adjuvant RT of 42-63 Gy is known to reduce local recurrence of STS after conservative surgery $[12,13]$. However, as shown in this study, RT for RIS results in a high rate of RT-related complications, which is inevitable in high-dose regions. Due to the rarity and the histologic diversity within RIS, it is difficult to analyze the efficacy of secondary adjuvant RT after curative resection of RIS. Extrapolating from sporadic STS, adjuvant RT should also be performed for RIS, but care is required in 


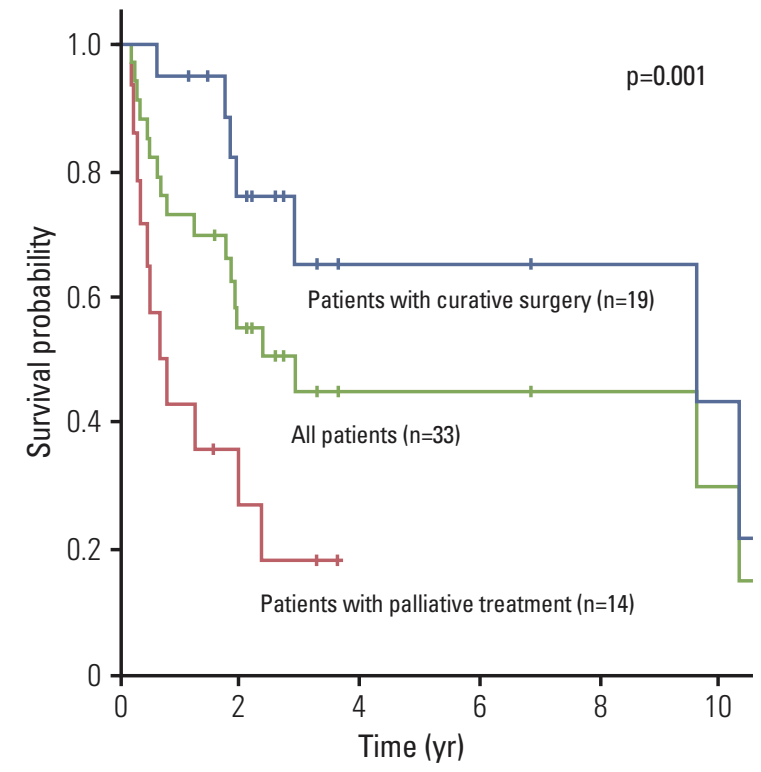

Fig. 2. Overall survival of patients with radiation induced sarcoma.

the process of consultation with the patient, RT planning, and prescription of the RT dose. Also, high-precision RT techniques with increased conformality, such as intensitymodulated radiation therapy (IMRT), should be used in such re-irradiation cases to minimize the toxicity.

The question of whether or not the use of IMRT, stereotactic body radiation therapy, or proton therapy would reduce RIS incidence is yet to be determined. From the follow-up observation data on atomic bomb survivors who showed no excess of sarcoma after exposure to radiation of a few Gy, we acknowledge that RIS is less likely to arise in very low-dose regions, and more likely to arise in intermediate- to highdose regions of above $5 \mathrm{~Gy}$, with some evidence of a doseresponse relationship [14,15]. However, as studies regarding RIS, including this study, lack data on the initial dose prescribed for the RIS lesion due to the loss or incompleteness of the topographic primary RT planning information, accurate dose-incidence evaluation is limited. Increased conformality with high-precision RT techniques would reduce the high-dose region, but integral doses in irradiated regions can be increased. In this context, Hall and Wuu [16] insisted that IMRT would double the incidence of secondary malignancy. On the contrary, in a risk estimation study, IMRT or proton therapy did not increase the risk of secondary cancer, but it was assumed that proton therapy reduced the incidence [17]. These findings should be demonstrated by clinical data in future studies.

In this study, four RIS patients misdiagnosed with bone metastasis underwent palliative RT before the biopsy, which is not an optimal treatment for RIS. Although rare, the possibility of RIS must be considered when malignancy is suspected in the previously irradiated region, and pathologic confirmation must be considered before the treatment.

In this respect, identifying predisposing factors for development of RIS could be of clinical concern. In the US Surveillance, Epidemiology and End Results analysis by Berrington de Gonzalez et al. [14], a higher standardized incidence ratio for subsequent primary sarcoma was observed for patients treated at a younger age than those treated at older ages. In addition, several studies reported multiplicative effects of radiotherapy and chemotherapy for the subsequent risk of sarcoma [18-21]. However, there is a limitation in explaining the occurrence of RIS with these clinical factors giving way

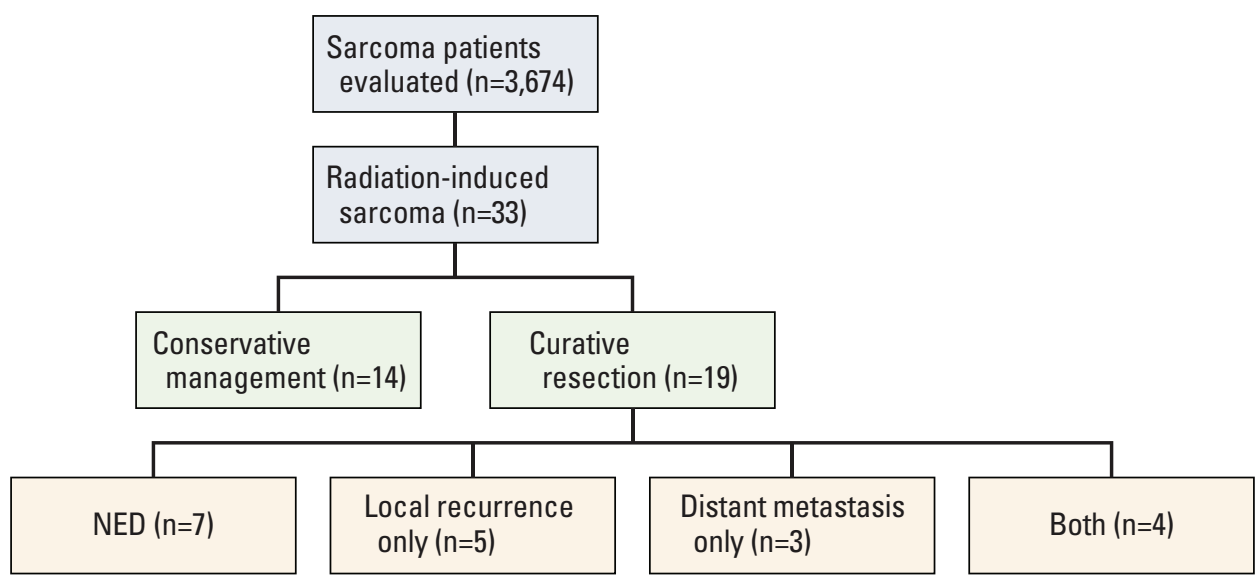

Fig. 3. Summary of treatment outcome of patients with radiation-induced sarcoma. NED, no evidence of disease. 


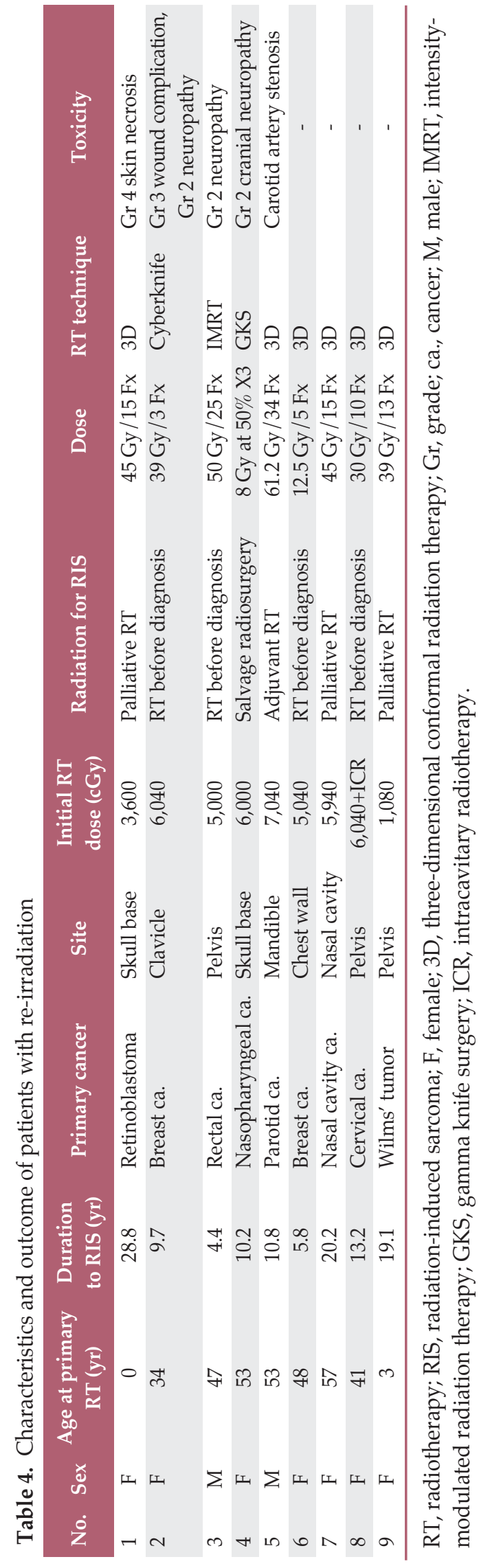

to the idea of possible genetic predisposition of a patient for development of RIS. Cha et al. [11] pointed out that in the early 2000s, there was no molecular or pathological marker that was helpful in accurately distinguishing RIS from spontaneous sarcoma, and little has changed since then. A study by Kacker et al. [22] found that MYC gene amplification was prevalent in RIS. On the other hand, the study by Kadouri et al. [1], investigating the role of p53 and BRCA mutation in RIS development, found a higher frequency of $B R C A$ mutation in RIS patients, but was insignificant, thus they concluded that BRCA mutation should not be considered when making the treatment decision. An in vivo study by Kansara et al. [23] reported evidence that RB1 is required for development of radiation-induced osteosarcoma. A study by Hadj-Hamou et al. [24] comparing the transcriptome of sporadic sarcoma with that of RIS showed 135 gene signatures which could differentiate RIS from sporadic sarcoma, which indicated mitochondrial dysfunction with a hallmark of chronic oxidative stress. However, studies on genetic factors related to RIS are still in their early stage. Future studies on the biological background of RIS should be conducted in two major parts: that on the genetic susceptibility to RIS to distinguish high-risk patients before RT, and that on the molecular "radiation signature" that can distinguish RIS from sporadic STS beyond Cahan's criteria $[9,14]$.

\section{Conclusion}

In this study, we found that $0.9 \%$ of the sarcoma patients in the registry of a large tertiary center met the RIS criteria. Although prognosis of RIS is poorer than that of sporadic STS, a surgical approach with curative intent is needed if possible, from which we can expect a favorable survival outcome, as seen in this study. Considering the aforementioned difficulties in RIS treatment, future study should focus on selection of RIS patient candidates using molecular markers before RT and should compare the risks and benefits of administering RT before the treatment. In addition, enhancing the precision of RT would contribute to the reduction of RIS or related complications in the future, but warrants further investigation.

\section{Conflicts of Interest}

Conflict of interest relevant to this article was not reported. 


\section{References}

1. Kadouri L, Sagi M, Goldberg Y, Lerer I, Hamburger T, Peretz T. Genetic predisposition to radiation induced sarcoma: possible role for BRCA and p53 mutations. Breast Cancer Res Treat. 2013;140:207-11.

2. Mark RJ, Poen J, Tran LM, Fu YS, Selch MT, Parker RG. Postirradiation sarcomas: a single-institution study and review of the literature. Cancer. 1994;73:2653-62.

3. Gladdy RA, Qin LX, Moraco N, Edgar MA, Antonescu CR, Alektiar KM, et al. Do radiation-associated soft tissue sarcomas have the same prognosis as sporadic soft tissue sarcomas? J Clin Oncol. 2010;28:2064-9.

4. Lagrange JL, Ramaioli A, Chateau MC, Marchal C, Resbeut M, Richaud P, et al. Sarcoma after radiation therapy: retrospective multiinstitutional study of 80 histologically confirmed cases. Radiation Therapist and Pathologist Groups of the Federation Nationale des Centres de Lutte Contre le Cancer. Radiology. 2000;216:197-205.

5. Bjerkehagen B, Smastuen MC, Hall KS, Skjeldal S, Smeland S, Fossa SD. Why do patients with radiation-induced sarcomas have a poor sarcoma-related survival? Br J Cancer. 2012; 106:297-306.

6. Dineen SP, Roland CL, Feig R, May C, Zhou S, Demicco E, et al. Radiation-associated undifferentiated pleomorphic sarcoma is associated with worse clinical outcomes than sporadic lesions. Ann Surg Oncol. 2015;22:3913-20.

7. Thijssens KM, van Ginkel RJ, Suurmeijer AJ, Pras E, van der Graaf WT, Hollander M, et al. Radiation-induced sarcoma: a challenge for the surgeon. Ann Surg Oncol. 2005;12:237-45.

8. Neuhaus SJ, Pinnock N, Giblin V, Fisher C, Thway K, Thomas JM, et al. Treatment and outcome of radiation-induced softtissue sarcomas at a specialist institution. Eur J Surg Oncol. 2009;35:654-9.

9. Cahan WG, Woodard HQ, Higinbotham NL, Stewart FW, Coley BL. Sarcoma arising in irradiated bone: report of 11 cases. Cancer. 1948;1:3-29.

10. Cho KH. The challenges faced by the Korean Society for Radiation Oncology in the national healthcare system in Korea. Int J Radiat Oncol Biol Phys. 2014;90:725-8.

11. Cha C, Antonescu CR, Quan ML, Maru S, Brennan MF. Longterm results with resection of radiation-induced soft tissue sarcomas. Ann Surg. 2004;239:903-10.

12. Pisters PW, Harrison LB, Leung DH, Woodruff JM, Casper ES, Brennan MF. Long-term results of a prospective randomized trial of adjuvant brachytherapy in soft tissue sarcoma. J Clin Oncol. 1996;14:859-68.
13. Yang JC, Chang AE, Baker AR, Sindelar WF, Danforth DN, Topalian SL, et al. Randomized prospective study of the benefit of adjuvant radiation therapy in the treatment of soft tissue sarcomas of the extremity. J Clin Oncol. 1998;16:197-203.

14. Berrington de Gonzalez A, Kutsenko A, Rajaraman P. Sarcoma risk after radiation exposure. Clin Sarcoma Res. 2012;2:18.

15. Rubino C, Shamsaldin A, Le MG, Labbe M, Guinebretiere JM, Chavaudra J, et al. Radiation dose and risk of soft tissue and bone sarcoma after breast cancer treatment. Breast Cancer Res Treat. 2005;89:277-88.

16. Hall EJ, Wuu CS. Radiation-induced second cancers: the impact of 3D-CRT and IMRT. Int J Radiat Oncol Biol Phys. 2003;56:83-8.

17. Schneider U, Lomax A, Timmermann B. Second cancers in children treated with modern radiotherapy techniques. Radiother Oncol. 2008;89:135-40.

18. Tucker MA, D'Angio GJ, Boice JD Jr, Strong LC, Li FP, Stovall $\mathrm{M}$, et al. Bone sarcomas linked to radiotherapy and chemotherapy in children. N Engl J Med. 1987;317:588-93.

19. Hawkins MM, Wilson LM, Burton HS, Potok MH, Winter DL, Marsden HB, et al. Radiotherapy, alkylating agents, and risk of bone cancer after childhood cancer. J Natl Cancer Inst. 1996; 88:270-8.

20. Le Vu B, de Vathaire F, Shamsaldin A, Hawkins MM, Grimaud E, Hardiman C, et al. Radiation dose, chemotherapy and risk of osteosarcoma after solid tumours during childhood. Int J Cancer. 1998;77:370-7.

21. Menu-Branthomme A, Rubino C, Shamsaldin A, Hawkins MM, Grimaud E, Dondon MG, et al. Radiation dose, chemotherapy and risk of soft tissue sarcoma after solid tumours during childhood. Int J Cancer. 2004;110:87-93.

22. Kacker C, Marx A, Mossinger K, Svehla F, Schneider U, Hogendoorn PC, et al. High frequency of MYC gene amplification is a common feature of radiation-induced sarcomas. Further results from EORTC STBSG TL 01/01. Genes Chromosomes Cancer. 2013;52:93-8.

23. Kansara M, Leong HS, Lin DM, Popkiss S, Pang P, Garsed DW, et al. Immune response to RB1-regulated senescence limits radiation-induced osteosarcoma formation. J Clin Invest. 2013;123:5351-60.

24. Hadj-Hamou NS, Ugolin N, Ory C, Britzen-Laurent N, SastreGarau X, Chevillard S, et al. A transcriptome signature distinguished sporadic from postradiotherapy radiation-induced sarcomas. Carcinogenesis. 2011;32:929-34. 